\title{
DEVELOPMENT OF 4 TESLA PERMANENT MAGNET
}

\author{
M. Kumada, T.Fujisawa, Y.Hirao, National Institute of Radiological Sciences, \\ 4-9-1 Anagawa, Inage, Chiba, 263-8555 Japan \\ M. Endo, M.Aoki, T.Kohda, Sumitomo Special Metals Co., Ltd., \\ 2-15-17,Egawa, shimamototyou, Mishima-gun, Osaka, 618-0013,Japan, \\ I. Bolshakova, R. Holyaka, Magnetic Sensor Laboratory, Lviv Polytechnic National University, \\ 1 Kotliarevsky Str., Lviv 79013, Ukraine
}

\begin{abstract}
Strong model dipole permanent magnetic for various kinds of accelerator applications is under development at NIRS. Field strength exceeding 4 Telsa in the magnet gap is achieved by an extended Halbach type magnet configuration. The field level is by more than factor of three stronger than a residual field of the material. This will open a new application to an accelerator of various kinds, from a very compact and large scale hadron collider magnet of easy to operate accelerator without power supply.
\end{abstract}

\section{INTRODUCTION}

The HIMAC has been the only ion accelerator dedicated to a cancer therapy. A successful treatment result of a cancer patients of about 1000 patients prompted to request to build more medical accelerator national wide. Second carbon ion synchrotron similar to the HIMAC has been constructed and is now commissioning in Hyogo prefecture and the irradiation to patients recently commenced. At the same time proton cyclotron and synchrotrons are also under construction at several other locations in Japan. Due to a lighter kinetic momentum, a size of proton accelerator is smaller than that of the carbon accelerator and less expensive in cost.

A rival of a charged particle accelerator is a $\mathrm{x}$-ray radiation therapy system if which X-ray is generated by a very compact linear accelerator. This accelerator is often called, a charming name of, gamma knife. Due to its drastic reduction in size and a cost of the gamma knife, it has been spreading to our society to a great extent.

Although a high-energy carbon ion accelerator is more effective than proton accelerator or gamma knife, the size was no trivial and the cost was not small as well. To reduce the size and the cost, necessary condition is to increase bending field strength of the dipole magnet. The standard magnetic field strength of the synchrotron magnet is 1.5 Tesla where at this level iron pole and yoke are already highly saturated.

It is well known that the saturation level of the DC magnet is higher than AC magnet. Two 3 Tesla proton cyclotrons have been manufactured and tested at MGH and Kashiwa cancer center. One of which has been in commissioning for a very long period and the other started therapy.
To generate higher than 3 Tesla in gap of magnet having an accelerator quality has been a challenging task in Russia by a high current of a few hundreds kilo-amperes of pulsed magnet system for about 15 years for an application of compact medical synchrotron. Their goal was not yet met at this moment though.

Exceeding field strength of 3 Tesla is possible by superconducting magnet. As a medical machine, it has a disadvantage of using helium or refrigerator system in a hospital where maintenance cost is a considerable amount.

Cyclotron or FFAG (Fixed Field Alternating Gradient) accelerator can be a medical treatment accelerator. As a compact machine, a combination of cyclotron as an injector and FFAG as a main therapy accelerator is a one possibility. After a study of a maximum attainable field strength by permanent magnet, we immediately noticed that generating field strength over 3 Tesla with a reasonable transverse size, is possible[1]

After subsequent extensive study, we discovered a method of even exceeding 4 Tesla is possible[2] by Neodyum, Boron iron material, NEOMAX, of Sumitomo Special Metal inc. where the field strength is stronger by more than factor of 3 than its residual field strength of about 1.3 Tesla. .

We like to emphasize that the application of this type of magnet is not limited to a compact medical accelerator but also to a large-scale accelerator such as Very Large Hadron Collider. The application to a storage ring as well as a beam transport is obvious.

\section{HOW TO GENERATE A HIGH FIELD STRENGTH BY PERMANENT MAGNET MATERIAL}

In most of permanent magnets, field strength is a few $\mathrm{kG}$ level. Question arises naturally, "Is there a theoretical limit generated by a permanent magnetic material? "

To answer this question, consider a simple magnetic circuit of a permanent magnetic material of length $1_{m}$ and gap height $d$ where width of an iron pole and iron yoke are assumed to be the same. Applying an ampere's law , the field strength $\mathrm{B}_{\mathrm{g}}$ at the gap is,

$$
B_{g}=\frac{l_{m}}{d+l_{m}} \mu_{0} H_{c}
$$


where $H_{c}$ is a coercive field and $B_{g}$ is a residual field of the magnetic material. In this ordinary configuration where magnetic material is combined with iron, an attempt to increase the gap field by increasing the length of the magnet portion compared with iron portion does not help much. In this equation, the gap field can not be exceeded $\mu_{0} \mathrm{H}_{\mathrm{c}}$ which is roughly the residual field of the material $\mathrm{B}_{\mathrm{r}}$. The typical residual field of the very strong material NEOMAX is 1.3 Tesla. Other rare earth material such as samarium cobalt is weaker by $10 \%$ or so. And that of Strontium Ferrite is $1 / 3$ of it. Thus 1.3 Tesla is the maximum field strength achievable in a conventional configuration.

There is an another approach. Klaus Halbach invented a novel configuration using a Rare Earth Cobalt magnet material which he called REC magnet. In his magnetic circuit, the direction of the magnet is rotated along a circular ring. The theoretical magnetic field inside an inner radius of the magnet given by Halbach is,

$$
B_{g}=\mathbf{B}_{r} l_{n}\left(\frac{r_{2}}{r_{1}}\right)
$$

where $r_{1}$ is an inner radius and $r_{2}$ is an outer radius of the REC magnet. Although a dependence of the field strength is rather insensitive, logarithmic to a ratio of an outer to an inner radius of a material, to a size of the magnet, there is no upper limit in this configuration. This equation, however, holds only true in a limited filed strength region. At very high field strength a demagnetization effect can not be neglected. With increasing field strength, the outer part of the magnet material generates a strong enough field to demagnetize inner part of the magnet material and a decrease of a magnetization which result in a decreasing field strength inside the magnet gap is inevitable. So far in most of the high field magnet the field strength is designed to be less than a residual field. Even in a REC high gradient Quadrupole permanent magnet of $250 \mathrm{~T} / \mathrm{m}$ developed by the author 16 years ago[3], trying to achieve the record strength, the pole tip field was designed at a residual field level.

We have invented a new configuration to overcome the problems associated above [2]. This is to introduce a saturated iron pole in a magnetic circuit of the permanent magnet. The role of an iron pole is namely threefold;

i) Use the iron as an equivalent of the magnetic material having stronger residual field.

ii) Use the iron to squeeze the flux of the permanent magnet material and let it go through the gap in a concentrated way(flux compression).

iii) Guide a flux nearby the pole so that the demagnetizing field becomes weak(flux guide).

Similar concept was adopted in superconducting cyclotron magnet at Michigan university[4].

\section{MODEL HIGH FIELD MAGNET}

In order to verify the above idea, a small model magnet is designed, constructed and the field strength and distribution are measured. The inner diameter of the magnet is $6 \mathrm{~mm}$ and the outer diameter is $200 \mathrm{~mm}$ and the length is $150 \mathrm{~mm}$.

This diameter is about half of the minimum gap of the world strongest high field DC conventional electromagnet of IBA cyclotron and is not at all surprisingly small even as a model magnet. A straight extension to a larger magnet gap is obvious by a simple scaling.

We have started from a Halbach type structure and modified it by introducing a saturated iron pole. The iron pole is placed near the gap so that high material of high magnetization is near by the gap. We like to call this configuration an Extended Halbach Structure. In addition to the strategy making use of the saturated iron in a permanent magnet structure, weakening the demagnetization effect was devised[2];

Two layer magnetic configuration was adopted to avoid demagnetization effect as much as possible: The material of the inner layer and the outer layer was chosen to be different, namely high $\mathrm{B}_{\mathrm{r}}$ material at the outer part of the magnet and high $\mathrm{H}_{\mathrm{c}}$ material at the inner part of the magnet is used.

The orientation of the magnetization of each element material of the inner layer is also modified to reduce the de-magnetization effect. This is due to our discovery that perpendicular component of the external field could demagnetize the material to an appreciable amount of degree

We assume that flux density in the gap, saturated iron pole and the permanent magnet material differ. It can be shown that the magnetic field strength $\mathrm{B}_{\mathrm{g}}$ in the gap is,

$$
B_{g} \approx \frac{\left[B_{r} l_{m}+\frac{l_{i}}{\mu} B_{s o}\right]}{d+\frac{l_{i}}{\mu^{*}} \frac{S_{g}}{S_{i}}+l_{m} \frac{S_{g}}{S_{m}}}
$$

where $\mathrm{S}_{\mathrm{g}}, \mathrm{S}_{\mathrm{i}}, \mathrm{S}_{\mathrm{m}}$ are cross sections of the effective gap, iron pole cross section and permanent magnet cross section., $\mu^{*}$ is a relative permeability of the iron respectively. The second term in the denominator and the second and third term in the numerator show our characteristic features of the design. The saturation field of the iron $\mathrm{B}_{\mathrm{s} 0}$ exceeds 2 Tesla, which is stronger than the magnetization of the permanent magnet material! Expensive permendule of slightly higher saturation magnetization can also be used as a pole tip material. We are currently studying an availability of higher magnetization material, which may be used in a next future model magnet to achieve a field strength exceeding 5 Tesla never conceived before in a history of permanent magnet

The compression factor of the flux is expressed as a ratio of the cross sections. In conventional permanent 
magnet with iron, the second term in the denominator can be neglected as the iron is not saturated. Following this new design principle, the model magnet is constructed. This is shown in Fig.1. The special Hall Generator Probes are developed at magnetic sensor laboratory of Lviv Polytechnic National University for an accurate field measurement of small aperture.

In spite of a pre-caution of minimizing the demagnetization effect, a reduction of the field strength of about $14 \%$ from the design value could not be avoided. The field strength was about 3.9 Tesla. Furthermore due to a strong magnetic force, a difficulty of assembly was encountered. Appreciable amount of quadrupole component was detected. After introducing a magnetic shimming to get a better field homogeneity, a peak local deviation less than $0.4 \%$ from the design distribution was achieved.

To increase the field strength above 4 Tesla, we made use of a temperature dependence of a permanent magnet. The magnet was cooled to minus 25 degree $\mathrm{C}$. The resulting observed field strength was 4.45 Tesla, the first permanent magnet exceeding 3 Tesla. (Previous record was 2.5 Tesla in a wiggler using the same material.) We are currently re-assembling the magnet to have better mechanical accuracy. Permendule will also be replaced with an iron pole to further increase the gap field strength.

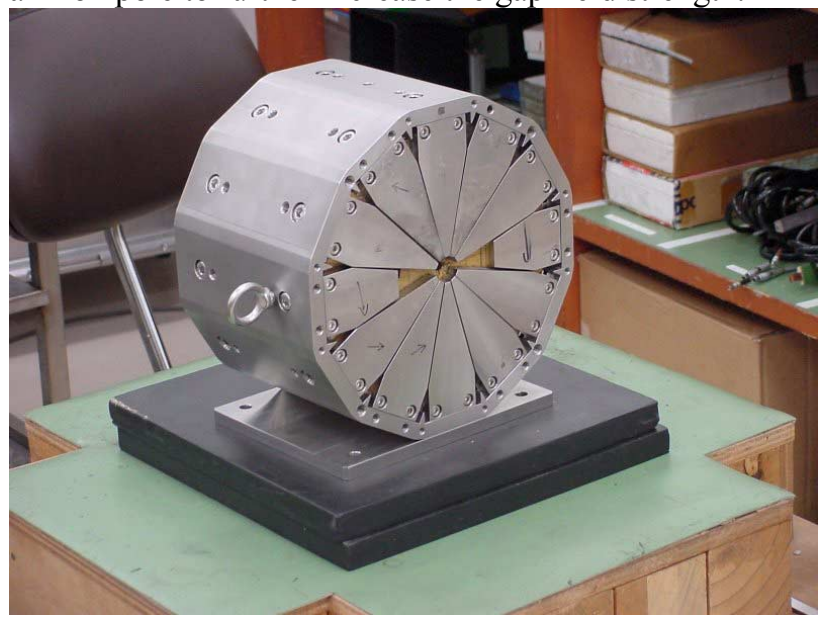

Fig.1 Assembled 4 Tesla permanent magnet

\section{APPLICATION OF HIGH FIELD PERMANET MAGNET}

The direct application of the strong permanent magnet is a FFAG magnet. In conventional FFAG there are least two types; a radial type FFAG and spiral type FFAG. The radial type is simple and consists of bending magnets of opposite directions aligned along an azimuth direction, which causes a loss in circumference length. The most compact spiral FFAG does not have this defect with a cost of complication. Currently we are studying a possibility of other type of FFAG, which will be presented in a forthcoming paper.

The expected advantage of the FFAG is its possible high repetition rate of ion beam acceleration. As the medical accelerators do not generally require high beam intensity, the high repetition feature of the FFAG is favored to make the magnet small and light weight.

The extremely lightweight is possible for a small gap FFAG. This feature makes it possible to place the accelerator vertically and even rotate the FFAG accelerator just like a rotating gantry to direct a carbon ion beam from various directions to the patients under irradiation.

When the main accelerator of several hundreds $\mathrm{MeV} / \mathrm{u}$ is made compact, the injector of several $\mathrm{MeV} / \mathrm{u}$ must also be more compact. In the past, permanent magnet based cyclotron was constructed at LBNL. The project was terminated during the commissioning due to a termination of the funding [4]. AVF cyclotron made of high field permanent magnet is a possible candidate. Diameter of 2.5 Tesla cyclotron can accelerate proton equivalent energy of $12 \mathrm{MeV}$. Scaled down model magnet of this cyclotron magnet is now under design study in our group.

The high field permanent magnet is suitable for high energy hadron collider as well as a storage ring where the beam size is very small. A vertical gap size of VLHC is between $15 \mathrm{~m}$ and $20 \mathrm{~mm}$. The acceleration of the beam can be done either changing the field by a mechanical rotation or by FFAG accelerator. Field strength of 3 Tesla can be adequately designed with a diameter of 300$\mathrm{mm}$ outer size with a reasonable cost. This option should be compared with 2 Tesla low field version of the VLHC.

Other application of the high field permanent is a compact FFAG synchrotron radiation ring. In this scheme, the electron beam is directly injected and accelerated and stored in the permanent magnet FFAG. Due to the high magnetic field, a wiggler magnet can be omitted. Medical application to Rotating gantry or high field MRI magnet is also promising.

\section{CONCLUSION}

We showed how to generate field strength much beyond the residual field of the permanent magnet material. A model magnet was constructed and the field strength of 4.45 Tesla was demonstrated and field shimming was applied to get designed field distribution by an Extended Halbach structure where saturated iron pole is incorporated. This model high field magnet could be scaled up to a large magnet such as FFAG magnet. Cyclotron magnet and Very Large Hadron Collider.

\section{REFERENCES}

[1] M.Kumada patent under submission.

[2] M.Kumada and T.Kohda, patent under submission

[3] M.Kumada et.al Magnet Technology conference, 1985, Zurich.

[4] H.Blossser (MSU), private communication

[5] A.Young (LBNL), private communication 